\title{
ACOUSTIC EMISSION METHODS APPLIED TO AVALANCHE-FORMATION STUDIES*
}

\author{
By V.P. YEPIFANOV \\ (The U.S.S.R. Academy of Sciences, Institute of Mechanics Problems, Moscow, U.S.S.R.) \\ and V.P. KUZ'MENKO
}

(The U.S.S.R. Goskomgidromet, Central Asian Regional Research Institute, Tashkent, U.S.S.R.)

ABSTRACT. The relationship between the intensity of snow acoustic emission impulses and snow-cover stability is revealed by measuring the physical and mechanical properties of the snow cover in the starting zones of avalanches. This relationship is fundamental to the remote identification of an avalanche-hazard period.

In order to estimate the mechanical properties of a snow layer, a method of applying a rigid penetrometer equipped with a piezo-electric accelerometer is used. The viscosity coefficients of snow under destruction and the specific energy of destruction are determined. The overall effect of different elements of destruction is assessed using both structural investigations and acoustic methods (acoustic emissions).

\section{INTRODUCTION}

The basic concept of a weakened layer as the primary source of instability in the snow cover on a slope was used as an initial working hypothesis which determined the programme for the study of avalanche-formation conditions.

This paper presents a summary of investigations conducted at the field sites "Dukant" and "Chimgan" situated on the spurs of the western Tianshan, as well as at the site "Desna" near Moscow.

The high sensitivity of acoustic methods to changes in the structure of a material under deformation was a determining factor in the application of some of these methods to study snow-cover properties in an avalancheprone zone. The aim of the work was to show the possible acoustic methods applicable in the remote recognition of avalanche-hazard situations. A method of acoustic emission measurement is used together with a method using a penetrometer equipped with a piezo-electric accelerometer. The paper by St. Lawrence and Bradley (1977) demonstrated the relationship between acoustic emission through the snow cover and its stability on slopes. Sommerfeld $(1977,1982)$ and Gubler (1980) found a correlation between lowfrequency noise and snow-cover stability. Gubler used accelerometers inserted in the snow and demonstrated the starting time of natural avalanche activity. In contrast to Gubler (1980), we used wide-range microphones installed in the starting zone of an avalanche. The time of avalanche hazard was determined by simultaneous measurements of acoustic emissions in the avalanche starting zone and of the mechanical characteristics of a specific weakened layer. The solution of this problem was preceded by laboratory tests on the mechanical properties of snow and formulation of a complex approach, that is, recording not only the amplitude and number of impulses but also the frequency

* See Annals of Glaciology, Vol. 9, 1987, p. 246 for an extended abstract. characteristics of acoustic emission signals which are of some importance to the analysis. Let us first consider the various spectra of destruction.

\section{SPECTRA OF DESTRUCTION}

Figure 1 shows the spectra of impact interaction between a sphere and a snow slab for energy values (1) $1.8 \mathrm{~J}$, and (2) $2.6 \mathrm{~J}$, and for an impact interaction energy of $5.2 \mathrm{~J}$ the emission is represented by three phases (3), (4), and (5). The abscissa represents a logarithmic scale of frequency and the ordinate is the emission amplitude. The amplitude increases and the frequency composition of the spectra changes with an increase in impact energy,

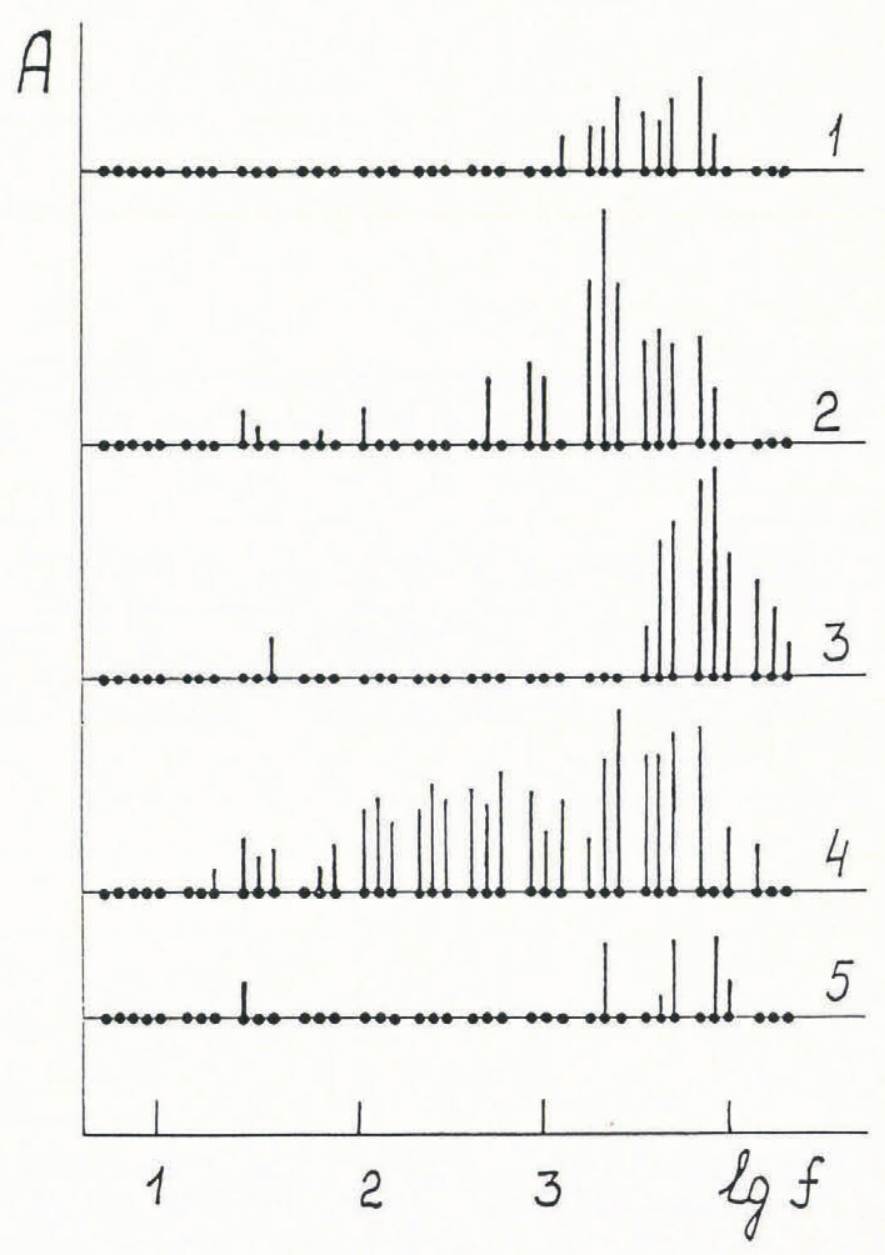

Fig. 1. The spectra of snow destruction. 
namely, the spectra are likely to reflect the intensity of destruction processes in snow.

The emission-intensity maxima are observed in the ranges of: I, $4-8 \mathrm{kHz}$; II, $2-2.5 \mathrm{kHz}$; III, $250-500 \mathrm{~Hz}$, and IV, $25 \mathrm{~Hz}$. Using model representations of harmonic oscillations (Voronina and Yepifanov, 1980) and considering the real sizes of the structural elements of snow, we can assess the degree of destruction corresponding to each of these four ranges. For example, the effective radius $R_{\mathrm{e}}=1.4 \mathrm{~mm}$ corresponds to the first high-frequency range, 4-8 kHz. This radius is the mean size of a neck. It can be determined that there are other degrees of destruction: II, crystallite destruction; III, collapse of the grain aggregates as structural formations; the IVth level refers to the process of dynamic interaction between the debris of structural formations. In the future, we proposed to use information on different scales of snow destruction to study processes preceding avalanche release.

Having related the characteristics of acoustic emission to the destruction of the elements of snow structure, we obtained a perspective method for remote determination of the process in the starting zone of avalanche formation; it is necessary to define the mechanical properties of the snow cover and to measure the processes of snow destruction separately from signals of acoustic emission.

\section{RESULTS OF DYNAMIC TESTS}

The mechanical properties of snow were determined using a penetrometer with a piezo-electric accelerometer (Yepifanov and Kudrya, 1985). Such penetrometers allow us to measure snow characteristics at any moment during collision. They were first used in ice tests (Yepifanov, 1985[a], [b]). Optimum masses and sizes of penetrometers, and initial collision velocities, were determined experimentally for various snow-cover conditions. Snow properties were assessed both in the vertical and horizontal directions.

The following equation is accepted as the initial case for stopping a free-falling rigid penetrometer:

$$
\ddot{X}(\tau)=g-\frac{1}{m} P(\tau)
$$

where $P$ is the response of snow, $\tau$ is time, $X$ is the depth of penetration, $m$ is the mass of the penetrometer, and $g$ is gravitational acceleration.

The voltage produced by a piezo-electric accelerometer is proportional to the force applied to the piezo-element; hence, to the penetrometer acceleration:

$$
\ddot{X}(\tau)=n C\left[U(\tau)-\frac{1}{\tau_{0}} \int_{0}^{\tau} \mathrm{d} \tau^{\prime} U\left(\tau^{\prime}\right)\right]
$$

where $n$ is the coefficient of transformation of the measuring system; $U$ is the voltage; $C, R$ are the capacity and electrical resistance of the measuring line, respectively; $\tau_{0}=R C$.

Figure 2 shows the dependence of the force $P, N$ (response of the snow) and mean stress at the sphere/snow interface $\sigma(\mathrm{MPa})$ (dashed line) on the depth of a sphere's penetration into the snow $X \times 10^{-3} \mathrm{~m} ;$ a smoothed oscillogram of impact interaction $U(\mathrm{mV})$ and the time of collision $\tau(\mathrm{ms})$ is also shown here.

The shape of the oscillogram and also the functions $P(X)$ and $\sigma(X)$ are determined by the collision process prevailing at a particular stage: (1) elastic deformation of grains and necks; (2) neck destruction; (3) elastic compression of air in pores; (4) viscous transport of grains, or (5) their collapse and densification. During stage 1, from the beginning of collision until the first extreme, which corresponds to brittle fracture, that is, snow hardness $\sigma_{\mathrm{T}}$, the initial structure would not change considerably and the penetration of a sphere into snow is due to elastic deformation. In our experiment $\sigma_{\mathrm{T}}=0.11 \mathrm{MPa}$.

The effective modulus $E=\partial \sigma / \partial X$ for stages $1-4$ of the relation equals $E_{1}=25 \times 10^{8} \mathrm{~Pa}, E_{2}=2.9 \times 10^{8} \mathrm{~Pa}$, $E_{3}=3 \times 10^{6} \mathrm{~Pa}$, and $E_{4}=20 \times 10^{8} \mathrm{~Pa}$, respectively; viscosity coefficients $n_{1}=25, n_{2}=41, n_{3}=73$, and $n_{4}=$

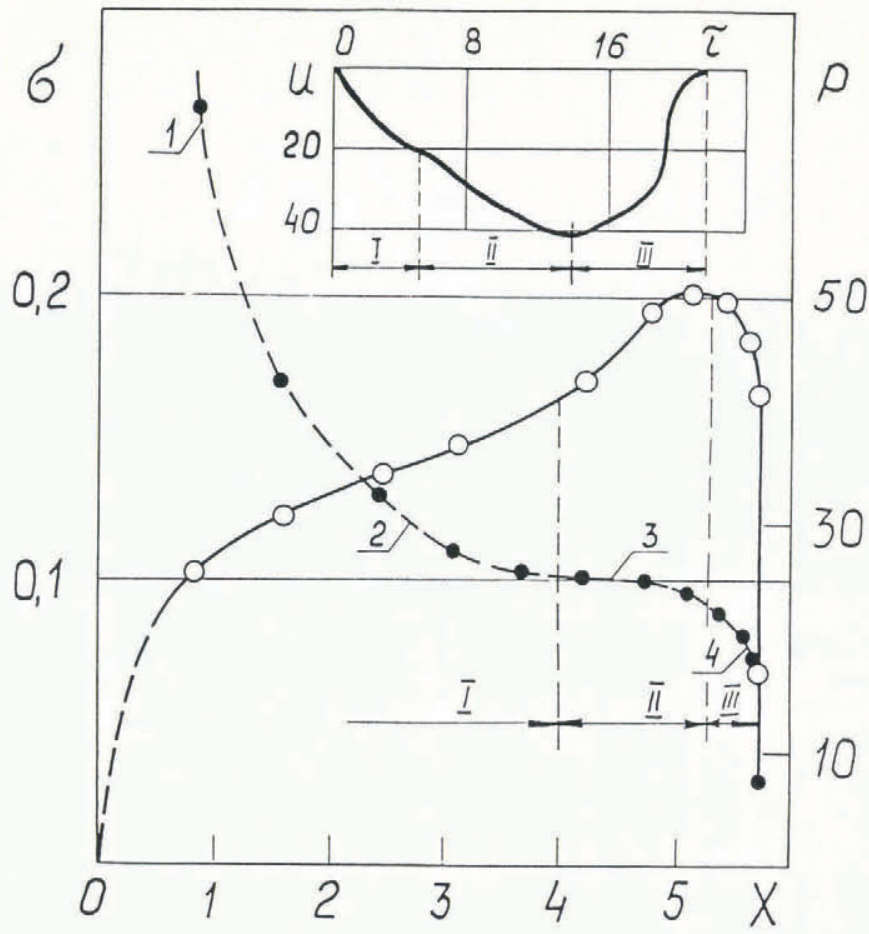

Fig. 2. A graph of impact-interaction loading.

$209 \mathrm{~Pa} \mathrm{~s}$. Time of relaxation is assessed by the relation $\tau=1 / \omega=n_{3} / E_{3}=2.4 \times 10^{-5} \mathrm{~s}$, and does not contradict the ice-relaxation time characterizing the elasticity of ice. Since $\omega=1 / \tau=2 \pi f$, the signals of acoustic emission may be expected to have a frequency of $f=6 \mathrm{kHz}$ during snow destruction. This fact has been confirmed experimentally and allows us to consider the computed values of $E$ and $\eta$ to be close to the real ones.

Thus, the penetration of a sphere into snow leads to the destruction of the initial snow structure and to the formation of a layer consisting of separate crystallites and their debris which, in turn, densifies. It has been shown that the mechanical properties of an undisturbed layer differ considerably from those of the densified snow. That is why, in order to define the characteristics of the undisturbed snow structure, we have considered only that part of the impact interaction process which precedes the collapse of the initial structure.

If we take the volume of a hole as an indicator of the degree of destruction and the energy losses in the first stage of a collision for the energy losses (defined by the $P(X)$ relationship), then the specific energy of destruction is $\varepsilon=6.6 \times 10^{4} \mathrm{~J} / \mathrm{m}^{3}$.

The true degree of destruction may be defined by changes in the mechanical properties of snow, e.g. hardness: greater compression corresponds to higher compaction, hence the greater hardness.

Figure 3 shows the dependence (1) of the $\sigma / \sigma_{0}$ ratio on time $\tau$ ( $\min )$. Hardness $\sigma$ along the track centre and hardness $\sigma_{0}$ of the undisturbed upper layer of snow cover were defined just after and during $1.5 \mathrm{~h}$ passage of a caterpillar-tracked vehicle, Buran. After passage of the vehicle hardness of the snow increased, approaching the threshold value. Over $45 \mathrm{~min}$, hardness of the compacted layer became about an order of magnitude higher than it was initially. Relaxation time $\tau_{2}=1.5 \times 10^{3} \mathrm{~s}$ is defined by the formula $\sigma / \sigma_{0} \sim f(\tau)$. This value characterizes the process of snow regaining, after passage of a vehicle, a new quasi-equilibrium state, i.e. plasticity.

The relationship $\sigma / \sigma_{\infty} \sim f(z)$ (Fig. 3, curve 3) gives us some idea about the course of hardness variation with snow depth $Z$ (cm) $90 \mathrm{~min}$. after the passage of Buran, hence about distribution of maximum stresses in the volume. Here, $\sigma_{\infty}$ is the hardness of a layer, where we failed to demonstrate a difference between the measured values before and after vehicle impact. In order to estimate the 


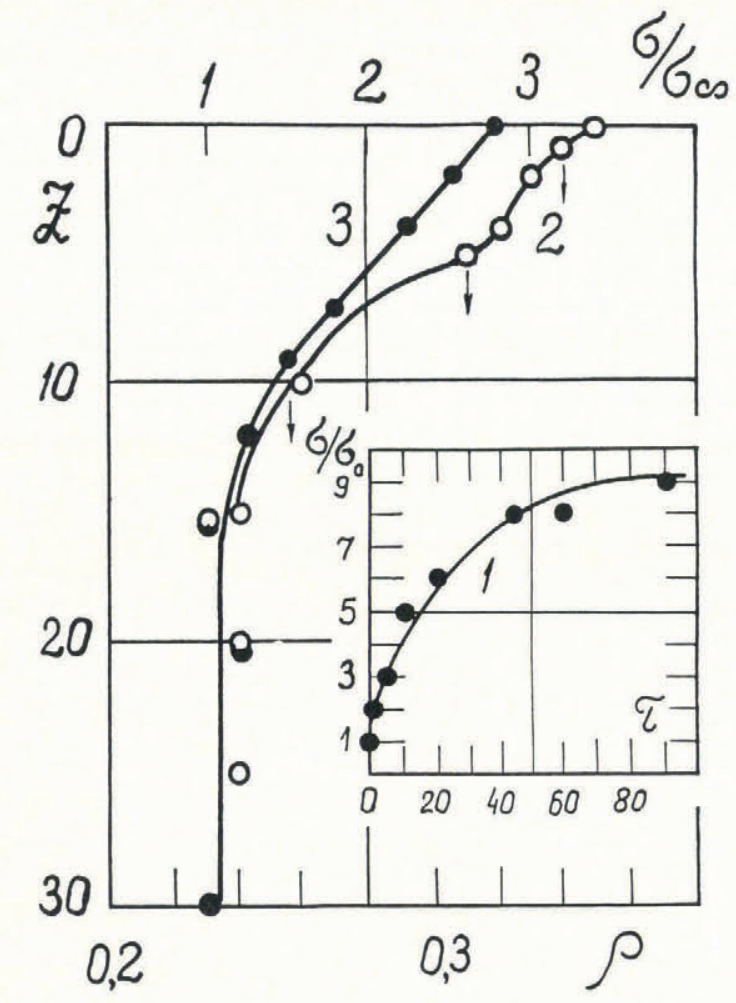

Fig. 3. Variations in snow properties after the passage of a vehicle.

hardness of every stratum in a snow layer, we have applied the method of making measurements in a pit. It appeared that the "compaction" cone did not reach the ground surface. Even at a depth of $10-15 \mathrm{~cm}$, it is difficult to observe after effects. So, it is quite simple to evaluate the true degree of destruction.

Snow density $\rho$ as a function of depth $Z$ was estimated at the same time as measuring its hardness. Curve 2 shows the same tendency to decrease as curve 3 , but hardness characterizes the mechancial properties of snow in a different way. Maximum hardness $\rho / \rho_{0}=2$ occurs at the surface of contact.

An average specific stress under a caterpillar-tracked Buran is calculated by the formula $P_{1}=K_{4}\left(M_{1} / S_{1}\right)$ when $K_{4}=2$, which is well known in mechanics. In our case, the coefficient of proportionality in Gerstner's law is $C_{1}=$ $P_{1} / X=4 \times 10^{3} \mathrm{~Pa} / \mathrm{m}$ when snow settling is $X=0.15 \mathrm{~m}$. Knowing the shape of the relationship $\sigma / \sigma_{\infty} \sim f(Z)$, which defines the stress in any layer normal to its axis, and having assumed that $\sigma_{\mathrm{T}} \sim P_{1}$, we can describe the snow behaviour under load (Ishlinskiy and Kondrati'yeva, 1950).

Hence, acoustic emission methods make it possible to assess the mechanical characteristics of snow and reveal the specific weakened stratum, as well as monitoring the processes in the snow cover for the determination of avalanche-formation conditions.

\section{FORECASTING AVALANCHE HAZARD}

We use the value $K=C / P$ as a criterion of avalanche hazard where $P$ is snow loading on a layer, and $C$ is the hardness or cohesion between particles in a weakened layer. This criterion has no specific advantages but in practice some scientists use it.

Figure 4 shows the dependence (1) of the stability parameter $K$, and (2) the acoustic frequency range, etc. on time $\tau(24 \mathrm{~h})$ for that section of the snow cover in the starting zone of any given avalanche-prone area which represents an erosion cone on the slopes of Mazardgan mountain (on the spurs of the Tianshan mountains). The mean angle of slope is $32-38^{\circ}$, and the exposure is from north to east-south-east. The slopes are covered by grass and sparse bushes; areas adjacent to the watershed lines are rocky.

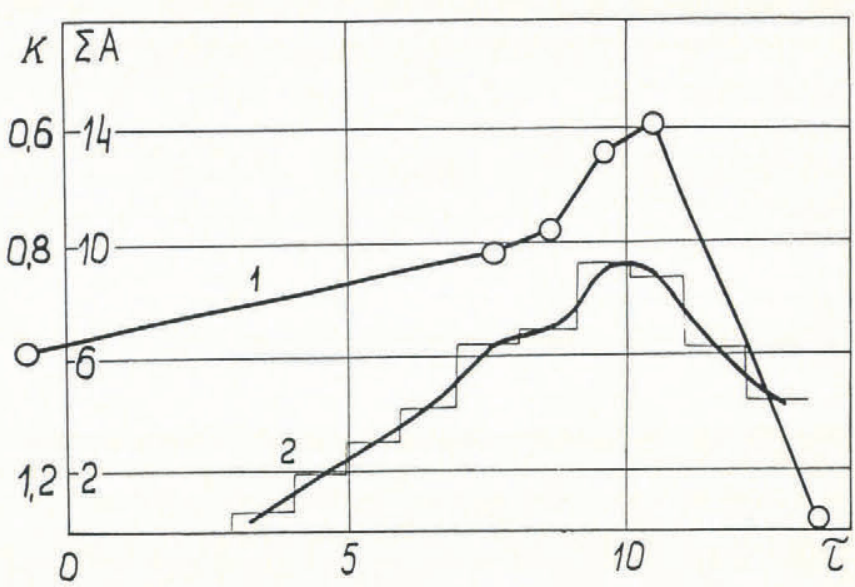

Fig. 4. Variation of the stability parameter $K$ and acoustic activity $\Sigma A$ in time $(24 \mathrm{~h})$ for avalanche-hazard slopes.

When $K=0.5$, the state of the snow cover on a slope is considered as an avalanche hazard. Difficulty in making pit measurements has compelled us to search for a remotesensing method for estimating snow-cover stability, e.g. using acoustic emission signals.

Intensification of the average activity of specific signals corresponds to a decrease in snow-cover stability. When the stability parameter has constant values or increasing ones, the intensity of acoustic emission decreases, reaching the background level; this is not in contradiction to the results of St. Lawrence and Bradley (1977), Sommerfeld (1977, 1982), and Gubler (1980).

The correlation found by considering the results of spectral analysis can be used to determine the stability of the snow cover on slopes; hence, we can foresee a way of using a remote-sensing method for monitoring avalanche hazard.

\section{CONCLUSION}

Thus, we have attempted to show there are good prospects for the use of acoustic emission methods in the study of a snow cover under stress and strain, and in forecasting the avalanche hazard. In particular, the process of snow destruction under conditions of compaction in various ways is investigated. The stages of destruction have been defined and the mechanical properties corresponding to these stages have been determined. We have assessed the distribution of stresses in the snow cover after passage of a caterpillar-tracked vehicle. The correlation between acoustic emission and the stability parameter of the snow cover on a slope has also been found.

\section{REFERENCES}

Gubler, H. 1980. Simultaneous measurements of stability indices and characteristic parameters describing the snow cover and the weather in fracture zones of avalanches. Journal of Glaciology, 26(94), 65-74.

Ishlinskiy, A. Yu., and Kondrati'yeva, A.S. 1950. [Turning rigid and pneumatic wheels on deforming ground.] In [Proceedings of the Meeting on Passage Capacity of Wheel and Caterpillar Vehicles on Virgin Terrain and Un-made-up Roads. Moscow, 25-29 May 1948.] Moscow, Izdatel'stvo Akademii Nauk SSSR, 68-88.

St. Lawrence, W., and Bradley, C. 1977. Spontaneous fracture initiation in mountain snow-packs. Journal of Glaciology, 19(81), 411-17.

Sommerfeld, R.A. 1977. Preliminary observations of acoustic emissions preceding avalanches. Journal of Glaciology, 19(81), 399-410.

Sommerfeld, R.A. 1982. A review of snow acoustics. Reviews of Geophysics and Space Physics, 20(1), 62-66. 
Voronina, I. Yu., and Yepifanov, V.P. 1980. [Acoustic investigations of structural variations in granite under axial compression.] Akusticheskiy Zhurnal, 26(3), 371-76.

Yepifanov, V.P. 1985[a]. Razrusheniye l'da pri udarnykh vzaimodeystviyakh [Ice fracture under impact loading.] Doklady Akademii Nauk SSSR, 284(3), 599-603.

Yepifanov, V.P. 1985[b]. [Some results of experimental studies of the mechanical properties of ice cover.] Akademia Nauk SSSR. Mekhanika Tverdogo Tela, 2, 182-91.

Yepifanov, V.P., and Kudrya, V.P. 1985. [A method for estimation of mechanical properties of visco-elastic materials under their impact interaction with an identor.] [The Plant Laboratory], 2, 64-66.

MS. received 10 January 1987 and in revised form 1 May 1987 